\section{Mini-Review}

\author{
Correspondence \\ Stephen Bornemann \\ stephen.bornemann@bbsrc.ac.uk
}

\title{
Unexpected and widespread connections between bacterial glycogen and trehalose metabolism
}

\author{
Govind Chandra, Keith F. Chater and Stephen Bornemann \\ Departments of Biological Chemistry and Molecular Microbiology, John Innes Centre, \\ Norwich Research Park, Norwich NR4 7UH, UK
}

\begin{abstract}
Glycogen, a large $\alpha$-glucan, is a ubiquitous energy storage molecule among bacteria, and its biosynthesis by the classical GlgC-GlgA pathway and its degradation have long been well understood - or so we thought. A second pathway of $\alpha$-glucan synthesis, the four-step GlgE pathway, was recently discovered in mycobacteria. It requires trehalose as a precursor, and has been genetically validated as a novel anti-tuberculosis drug target. The ability to convert glycogen into trehalose was already known, so the GlgE pathway provides a complementary way of cycling these two metabolites. As well as containing cytosolic storage glycogen, mycobacteria possess an outer capsule containing a glycogen-like $\alpha$-glucan that is implicated in immune system evasion, so the GlgE pathway might be linked to capsular $\alpha$-glucan biosynthesis. Another pathway (the Rv3032 pathway) for $\alpha$-glucan biosynthesis in mycobacteria generates a methylglucose lipopolysaccharide thought to be associated with fatty acid metabolism. A comparative genomic analysis was carried out to evaluate the occurrence and role of the classical pathway, the new GlgE pathway and the Rv3032 pathway across bacteria occupying very different ecological niches. The GlgE pathway is represented in $14 \%$ of sequenced genomes from diverse bacteria (about half as common as the classical pathway), while the Rv3032 pathway is restricted with few exceptions to mycobacteria, and the GlgB branching enzyme, usually presumed to be associated with the classical pathway, correlates more strongly with the new GlgE pathway. The microbiological implications of recent discoveries in the light of the comparative genomic analysis are discussed.
\end{abstract}

\section{Introduction: the classical view of the metabolism and functions of glycogen and trehalose}

This article first reviews recent discoveries in mycobacteria that have led to a substantial expansion in our understanding of the interlinked ways in which the carbohydrate storage compounds glycogen and trehalose are metabolized. We go on to show that a new pathway is unexpectedly widespread among diverse bacteria, and that the classical glycogen biosynthetic pathway is not as universal as was thought.

Glycogen is a largely soluble $\alpha$-linked glucose polymer (or $\alpha$-glucan) with $\sim 90 \% \alpha$-1,4-links in its backbone and $\sim 10 \% \alpha$-1,6-linked branches (reviewed by Preiss, 2009). Glycogen can comprise hundreds of thousands of glucose units and is generally synthesized in bacteria when there is an excess of carbon over another nutrient that limits growth (reviewed by Preiss \& Romeo, 1989). As a carbon/

Supplementary bioinformatic details (including Supplementary Table S1, listing the genes used in the reciprocal BLAST searches) and output (Supplementary Fig. S1, showing the presence of glucan-related genes within sequenced microbial genomes) are available with the online version of this paper. energy storage molecule, it can make up $60 \%$ of cell dry weight; it enhances the survival of cells (e.g. Escherichia coli) and it transiently accumulates prior to the onset of sporulation (e.g. in Bacillus cereus) or the production of exopolysaccharides (e.g. in Streptococcus mutans, which contributes to the formation of dental caries). Historically, bacteria have been considered to synthesize glycogen using the classical GlgC-GlgA pathway (Fig. 1) (reviewed by Preiss, 2006). This involves generating an activated glucose nucleotide diphosphate from glucose 1-phosphate by the action of nucleotide diphosphoglucose pyrophosphorylase $(\mathrm{GlgC})$, and its subsequent polymerization by glycogen synthase $(\mathrm{GlgA})$, to generate the linear glucan. This is turned into glycogen by the branching enzyme (GlgB)mediated transfer of non-reducing-end oligoglucans to the 6-position of residues within a chain to generate sidebranches. Throughout prokaryotes, glycogen has always been considered to be degraded by the highly conserved enzyme glycogen phosphorylase (GlgP) together with the debranching enzyme (GlgX), to generate glucose 1phosphate, which feeds readily into primary metabolism.

The other well-known abundant bacterial storage carbohydrate, the disaccharide trehalose, is both an energy 


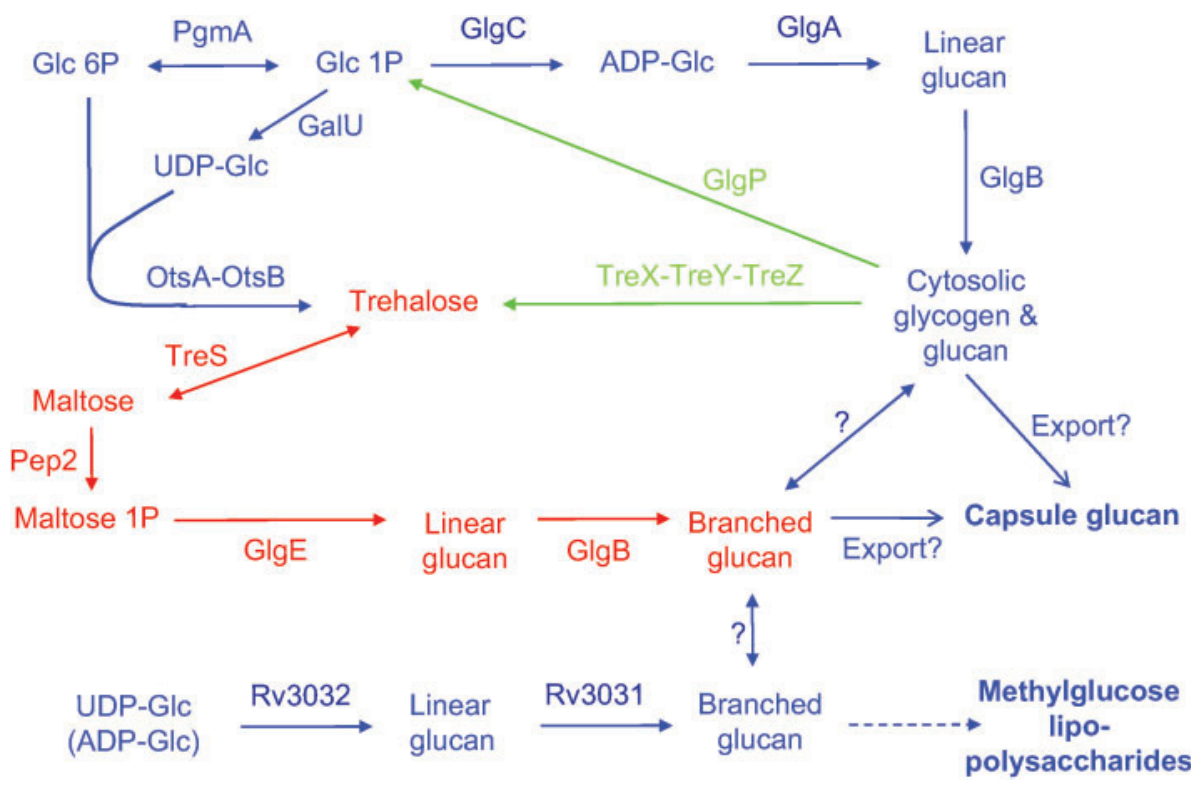

Fig. 1. A new view of bacterial glucan pathways. GlgC and GlgA are central to the classical glycogen pathway. The Rv3032 pathway is associated with methylglucose lipopolysaccharide biosynthesis. The newly identified GlgE pathway (red) may contribute to cytosolic glycogen, capsular glucan and/or methylglucose lipopolysaccharide biosynthesis - it is not yet clear which. Glucans are known to be degraded (green) either to glucose 1-phosphate by GlgP or to trehalose by TreX-TreY-TreZ (also known as GlgX-GlgY-GlgZ), generating potential intermediates for glucan reassembly or other metabolic purposes.

store and a stress-protectant, helping bacteria to survive desiccation, cold and osmotic stress (reviewed by Argüelles, 2000). Classically, trehalose ( $\alpha, \alpha-1,1$-linked diglucose) is synthesized in bacteria from glucose phosphate intermediates via trehalose 6-phosphate, using the GalU-OtsA-OtsB system (reviewed by Elbein et al., 2003). Trehalose can constitute more than $10 \%$ of cellular dry weight, and can be the major storage carbohydrate in specialized developmental states such as spores and the bacteroids central to the Rhizobium-legume symbiosis. In mycobacteria (and the related corynebacteria), trehalose has attracted particular attention because of its incorporation into cell wall mycolic acids, which are involved in pathogenesis and immune system evasion (reviewed by Takayama et al., 2005). The degradation of trehalose to glucose has been relatively little studied, in part because trehalases appear to be poorly conserved (Carroll et al., 2007).

The main theme of this minireview is the extent to which recent results have revealed more of the intimate and complex interplay of glycogen and trehalose metabolism in diverse bacteria. A first hint of such interplay was the discovery 15 years ago of the TreY-TreZ pathway in Sulfolobus acidocaldarius (Fig. 1; Maruta et al., 1996). In this pathway, TreY inverts the reducing-end glucosyl residue of $\alpha-1,4$ glucan to form an $\alpha, \alpha-1,1$-linked non-reducing trehalosyl disaccharide end, which yields free trehalose when it is cleaved from the glucan chain by TreZ. This process may be aided by glycogen debranching enzyme, TreX (also known as GlgX). Also in the 1990s, another enzyme, trehalose synthase (TreS) from Pimelobacter sp. R48, was discovered that could convert simple $\alpha$-1,4-linked diglucose (maltose) to trehalose (Fig. 1; Nishimoto et al., 1996).

\section{The Rv3032 route: the first non-classical pathway to bacterial $\alpha$-glucan}

The methylglucose lipopolysaccharides of mycobacteria typically comprise $\sim 20$ glucose units with an $\alpha$-1,4-linked backbone, $10 \% \alpha-1,6$ branching and extensive acylation (reviewed by Jackson \& Brennan, 2009). Despite such molecules being described 45 years ago (Lee, 1966), their role is not yet certain. They appear to be involved in chaperoning or regulating fatty acid biosynthesis within the cytosol by forming complexes with the products of fatty acid synthases. Recent studies in Mycobacterium tuberculosis have shown that a paralogue of GlgA, Rv3032, has $\alpha$ - $(1,4)$ glucosyltransferase activity that is associated with the generation of these specialized glucans (Kaur et al., 2009b; Stadthagen et al., 2007). Preliminary experiments suggested that Rv3032, unlike bacterial GlgA, might use UDP-glucose as well as ADP-glucose as the donor (Stadthagen et al., 2007). Branches are predicted to be introduced into the glucan by the branching enzyme Rv3031 (Stadthagen et al., 2007).

\section{GlgE mediates a new non-classical route to bacterial $\alpha$-glucan}

A third pathway to bacterial $\alpha$-glucans has recently been discovered in M. tuberculosis (Kalscheuer et al., 2010) and 
identified in part in Mycobacterium smegmatis (Elbein et al., 2010). Rather than using a monosaccharide nucleotide diphosphate, the novel maltosyltransferase GlgE uses a disaccharide phosphate, $\alpha$-maltose 1 -phosphate, as the building block to extend glucan chains. This is reflected in GlgE belonging to the GH13 family rather than the GT4 family to which Rv3032 and GlgA belong, as defined by the CAZy database (http://www.cazy.org/) (Cantarel et al., 2009). A GlgB enzyme introduces branches reminiscent of the classical pathway. Remarkably, maltose 1-phosphate is generated from trehalose, in two steps: TreS isomerizes trehalose into maltose (Pan et al., 2004); and a maltokinase Pep2 (also known as Mak1) phosphorylates the maltose using ATP (Jarling et al., 2004). Trehalose synthase TreS was originally assumed exclusively to generate trehalose from maltose in vivo (Elbein et al., 2003), but the discovery of the GlgE pathway has now shown that the reverse reaction is also physiologically important (Kalscheuer et al., 2010). While it is possible that the source of trehalose for the GlgE pathway could be via the de novo GalU-OtsAOtsB route, the alternative (TreX)-TreY-TreZ route allows for facile $\alpha$-glucan recycling. The latter route may be physiologically relevant because a role for GlgE in glycogen cycling was suggested by previous genetic studies (e.g. Belanger \& Hatfull, 1999).

\section{The correct operation of the non-classical pathways is important for mycobacterial growth, survival and pathogenicity}

Blocking either of the Rv3032 or GlgE pathways (by disrupting Rv3032 or treS, respectively) has no effect on the growth of M. tuberculosis (Kalscheuer et al., 2010). However, knocking out both is synthetically lethal. This implies that the pathways share an essential structurally or functionally related $\alpha$-glucan product. In other words, the pathways are at least partially redundant. In contrast, the disruption of both $g \operatorname{lgC}$ and Rv3032 is not lethal.

However, another $\alpha$-glucan needs to be considered. The extracellular capsule of mycobacteria contains an $\alpha$-glucan similar to glycogen that is implicated in the evasion of the mammalian immune system rather than in energy storage (Dinadayala et al., 2008; Gagliardi et al., 2007; Kaur et al., 2009a; Sambou et al., 2008). Disruption of Rv3032 has no effect on capsular glucan levels, implying that the classical pathway or the GlgE pathway is responsible for its biosynthesis. Surprisingly, disruption of either $g l g C$ or $g l g A$ from the classical pathway does not give clear answers and indeed gives different phenotypes. For example, disruption of $g l g C$ leads to both glycogen and capsular glucan levels being lowered, presumably due to lower ADPGlc levels. In contrast, disruption of $g l g A$ leads to only capsular glucan levels being lowered and an impairment of the mutant's persistence in mice, implying a link between GlgA, capsular glucan and immune system evasion (Sambou et al., 2008). The role of the GlgE pathway in capsular glucan biosynthesis and the complex interplay between the $\alpha$-glucan pathways has evidently yet to be fully deconvoluted.

Unexpectedly, disruption of $g l g E$ in M. tuberculosis is lethal in vitro and in a mouse model (Kalscheuer et al., 2010). Lethality is not due to the absence of the pathway product but rather to the accumulation of the GlgE substrate, maltose 1-phosphate, to presumably toxic levels. Maltose 1-phosphate accumulation and lethality are also observed with a $g l g B$ mutant (Kalscheuer et al., 2010; Sambou et al., 2008). Thus, both GlgE and GlgB have been genetically validated as novel anti-tuberculosis drug targets with a novel killing mechanism, although the former is the more attractive therapeutic target because $\mathrm{GlgB}$ homologues exist in mammals.

\section{Comparative genomics indicates considerable importance for the non-classical pathways across all bacterial classes but not Archaea}

Before being biochemically defined in mycobacteria (Kalscheuer et al., 2010), the GlgE pathway genes were first shown to be present in Streptomyces coelicolor (Schneider et al., 2000), while the Rv3032 pathway appeared to be confined to mycobacteria (Stadthagen et al., 2007) and their close relatives such as Nocardia species (Pommier \& Michel, 1986). This raises the question as to how widely the two non-classical pathways are distributed. For the purposes of this article, we therefore carried out comparative genomic analyses. We first made reciprocal BLAST searches of over 1000 complete bacterial and archaeal genomes using M. tuberculosis and S. coelicolor genes of $\alpha$-glucan metabolism as the query (for full technical details see the supplementary material available with the online version of this paper). In order to avoid bias towards Gram-positive gene sequences, we included $E$. coli genes as queries whenever they were known to exist (e.g. the classical glycogen pathway genes).

Unexpectedly, $g l g E$ was present in $21 \%$ of all completely sequenced bacterial genomes (including plasmids, where present), though only in one archaeal genome. When the distribution of the four genes of the glgE pathway (treS, pep2, $g \lg E$ and $g \lg B$ ) was assessed, it turned out that the whole $g l g E$ pathway was present in $14 \%$ of genomes analysed, while $36 \%$ of bacterial and $81 \%$ of archaeal genomes had none of the four genes (See Fig. 2 for absolute numbers of occurrences).

The organisms possessing the GlgE pathway mostly had large, GC-rich genomes and complex life styles, and included many that interact with plants. Those lacking these genes were often animal pathogens or had small genomes. The entire GlgE pathway was particularly well represented in actinomycetes, which include high-GC Streptomyces, Mycobacterium and Corynebacterium species (Supplementary Fig. S1), while low-GC Gram-positives, such as Clostridium and Bacillus species, tended to lack at least pep2 and glgE. In contrast, most Gram-positives had 


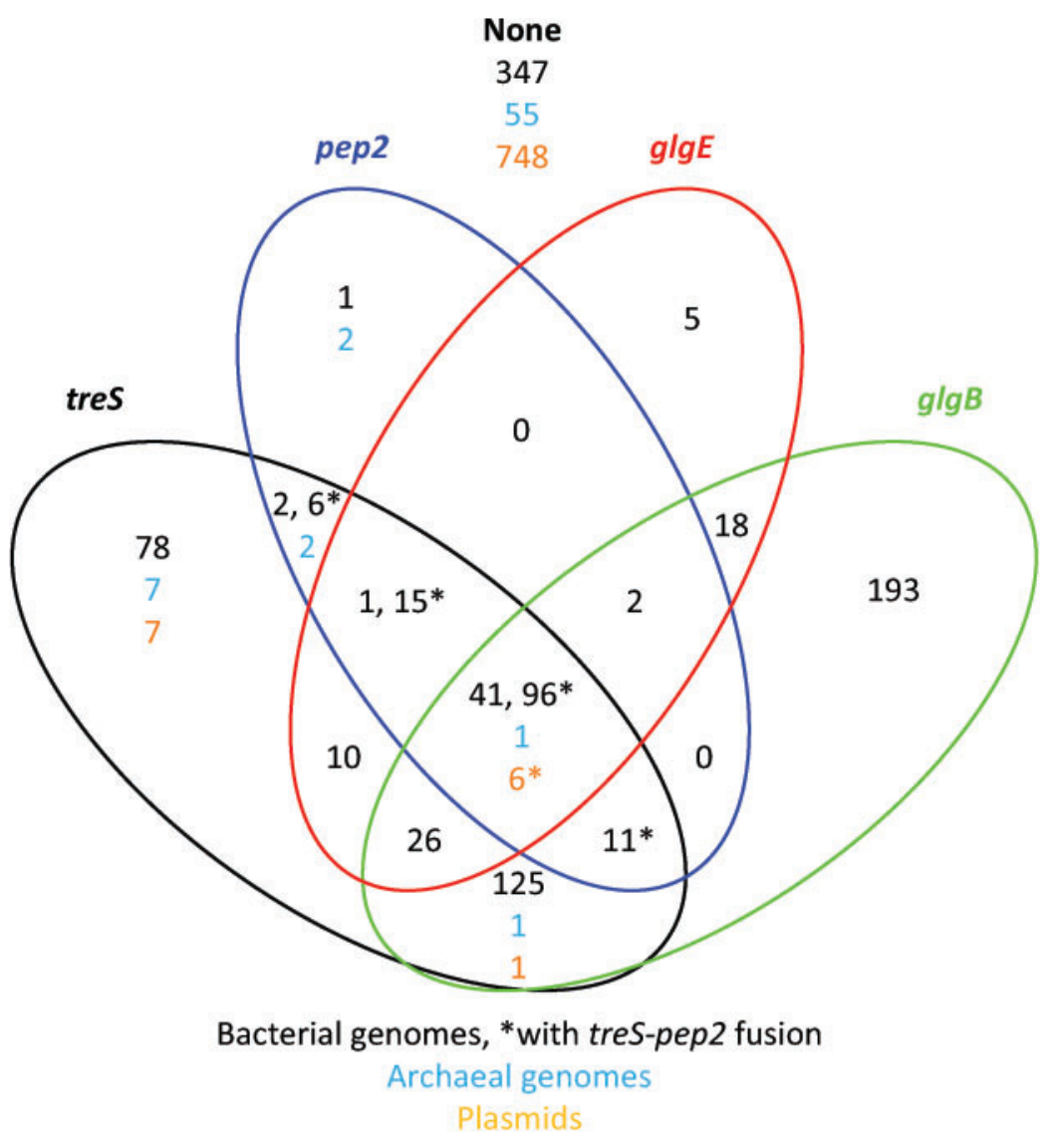

\begin{abstract}
Fig. 2. Venn diagram showing the occurrence of the treS, pep2, glgE and $g / g B$ genes within complete genomes and plasmids from bacteria and archaea. Of 1045 genomes, 977 were bacterial (black) and 68 archaeal (blue). A total of 762 plasmids were also included in this analysis (orange). The six co-occurrences of all four GlgE pathway genes on plasmids were associated with Rhizobium or Ralstonia species. Asterisks refer to occurrences where the treS and pep2 genes are fused. 'None' refers to the number of genomes and plasmids that have none of the GlgE pathway genes. See supplementary material for details.
\end{abstract}

the classical glycogen pathway genes. All four GlgE pathway genes were found sporadically in Gram-negative bacteria, including several Alphaproteobacteria (e.g. in Rhizobium, Rhodopseudomonas, Rhodobacter, Agrobacterium and Methylobacterium species), many Betaproteobacteria (especially in Burkholderia and Bordetella species), few Gammaproteobacteria (with the notable exceptions of xanthomonads and pseudomonads) and very few Deltaproteobacteria (but notably in Myxococcus xanthus). Other Gram-negatives such as Epsilonproteobacteria and Cyanobacteria tended not to have all four GlgE pathway genes. Among Gram-negatives, most Gammaproteobacteria and some Alphaproteobacteria but few others possessed the classical glycogen pathway genes. The only archaeal species with all four GlgE pathway genes was Picrophilus torridus DSM 9790, in which they were clustered. A BLASTN analysis did not provide evidence for the horizontal transfer of the cluster from the kingdom Bacteria. Most archaeal species, including $P$. torridus, lacked the genes for the classical glycogen pathway.

The GlgA pathway (as defined by the $g \lg A$ and $g \lg C$ genes) was roughly twice as common as the GlgE pathway and, as expected, the most common $\alpha$-glucan biosynthetic pathway (Fig. 3a). Reciprocal BLAST searches using Rv3032 alone as the query seemed to suggest that this gene was as common as $g l g A$. However, carbohydrate-active enzymes can evolve diverse functions with remarkably little sequence diversification
(Cantarel et al., 2009). In order to ascribe function to Rv3032 homologues more robustly, the co-occurrence of two additional genes thought to be required for the Rv3032 pathway was determined (Rv3030, a putative $S$-adenosylmethionine-dependent methyltransferase gene, and Rv3031, a putative branching enzyme gene) (Fig. 3a). This analysis confirmed that the Rv3032 pathway was restricted to some actinomycetes: mycobacteria, rhodococci, Nocardia farcinica IFM 10152 and Actinosynnema mirum DSM 43827. The three defining Rv3032 pathway genes were always clustered when they co-existed, and both other $\alpha$-glucan biosynthetic pathways were also usually present. Thus methylglucose lipopolysaccharides appear indeed to have a specialized role that is confined to very few bacteria. Somewhat surprisingly, $60 \%$ of bacteria and archaea had none of the three $\alpha$-glucan biosynthetic pathways.

\section{Clustering and fusion of GlgE pathway genes}

The pep2 and $g l g E$ genes rarely occurred in the absence of the other two genes of the glgE pathway (Fig. 2). Indeed, pep2 and treS were more frequently fused than separate, often leading to their underannotation as just one or the other. Thus, pep 2 and $g l g E$ are rather specifically associated with the $g l g E$ pathway. In contrast, both $\operatorname{tre} S$ and $g \lg B$ often occurred, on their own or together, without the other two 
(a)

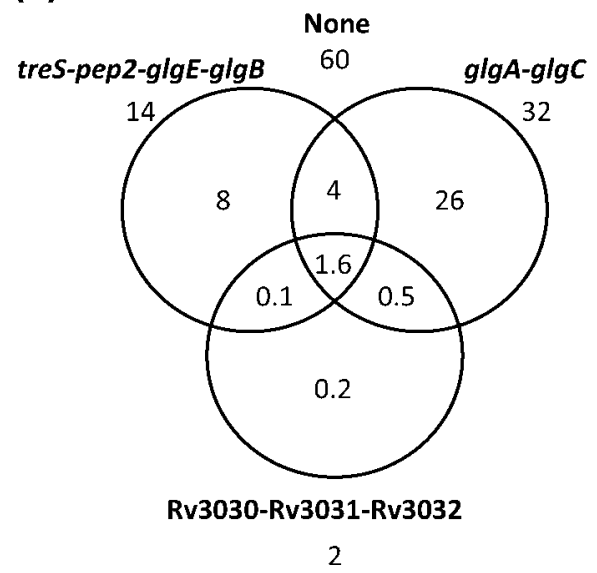

(c)

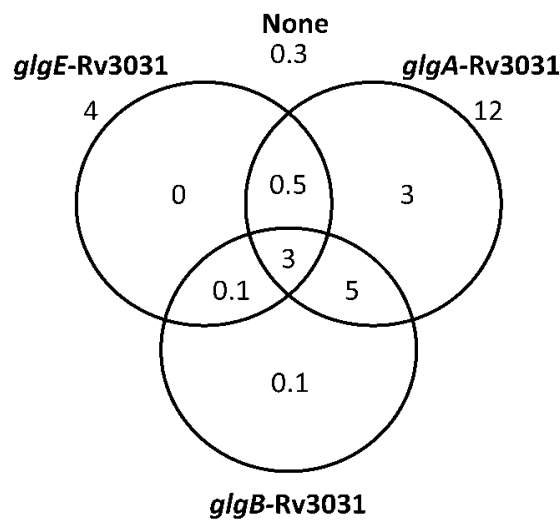

8

\section{(e)}

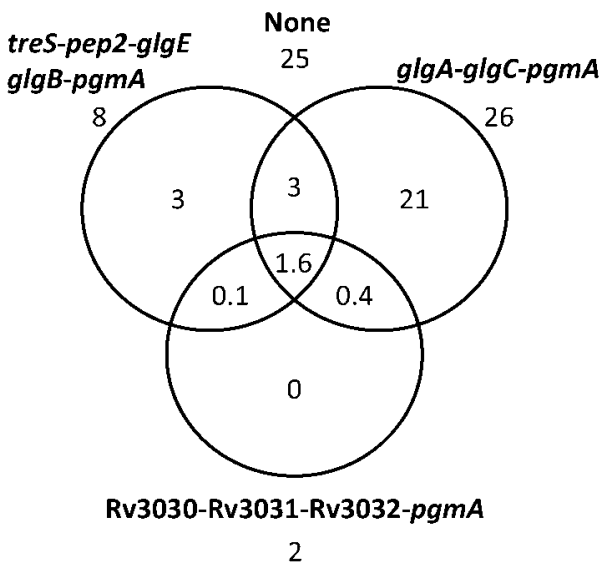

(b)

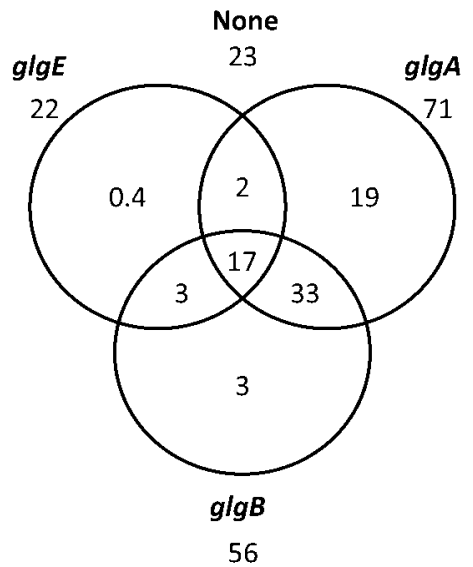

(d)

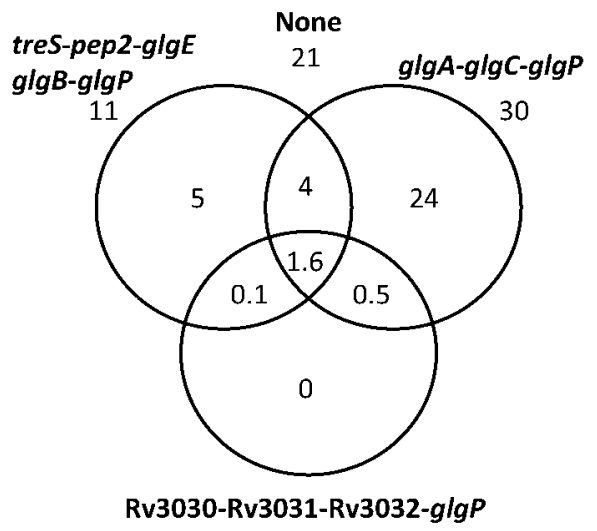

2

Fig. 3. Venn diagrams correlating the co-occurrence of genes encoding glucan biosynthetic and degradative enzymes. All values are expressed as a percentage of 1045 genomes analysed. (a) Genes defining the GlgA, GlgE and Rv3032 pathways (plasmids were devoid of these glucan pathway genes except for six occurrences of the GlgE pathway genes that amount to only $0.6 \%$ of genomes; see Fig. 2); (b) $g / g E, g l g A$ and $g / g B$; (c) $g / g E, g l g A$ and $g / g B$ (i.e. those in panel b) co-occurring with Rv3031; (d) GlgA, GlgE and Rv3032 pathway genes (i.e. those in panel a) co-occurring with glgP; and (e) GlgA, GlgE and Rv3032 pathway genes (i.e. those in panel a) co-occurring with pgmA. 'None' refers to the number of genomes that have none of the relevant genes. Numbers next to a set refer to the total percentage associated with that set. See supplementary material for details. 
genes. This may reflect roles in other contexts, such as the conversion of maltose to trehalose, and the GlgB-mediated branching of glucan generated by the classical pathway.

The clustering of the $g l g E$ pathway genes was assessed in each genome. Where all four functions were present, four alternative arrangements were found. If treS and pep2 were fused, the genes always formed a single operon-like cluster (Fig. 4a). This was the most common arrangement. When all four genes were present without a fusion they were equally likely to be in any one of three arrangements: fully clustered (Fig. 4b); in well-separated $\operatorname{glgE}-g \lg B$ and treSpep2 pairs (Fig. 4c); or substantially unclustered. When one of the functions was absent and treS and pep2 were fused, the cluster was broken up about one-third of the time. The genes were never clustered when only three genes were present with no fusion. Overall, the tendency of the genes to cluster, either altogether or as two pairs, implies the existence of operons allowing their co-ordinated expression. Genetic evidence of an operon structure was provided for the two copies of the cluster in S. coelicolor (Schneider et al., 2000).

\section{Frequent association of $g / g B, g l g P$ and both trehalose biosynthetic pathway genes with the GIgE pathway genes}

The GlgE, GlgA and Rv3032 $\alpha$-glucan pathways are all completed by the action of a branching enzyme. To date, GlgB has always been assumed to be associated with the GlgA pathway, because of the proximity of their genes in the well-characterized $g \lg P A C X B$ cluster in $E$. coli. However, $g l g B$ was surprisingly absent from $30 \%$ of the genomes that possessed $g l g A$ (Fig. 3b). Furthermore, $g l g B$ was separated (by $\geqslant 10$ genes) from $g l g A$ in $51 \%$ of cases where they coexist, making the clustering of the E. coli glg genes almost an exception rather than a rule. Unexpectedly, $g l g B$ was present in most $(89 \%)$ glgE-containing genomes, and was usually clustered with $g \lg E$ (in $80 \%$ of cases where they coexist). Only $8 \%$ of genomes had two copies of $g \lg B$ according to reciprocal BLAST searches, reducing to $3 \%$ if

(a)

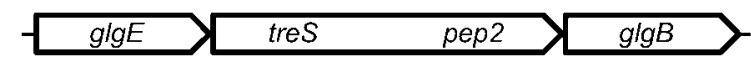

(b)

glgE $>$ tres $>$ pep2 $>$ glgB

(c)

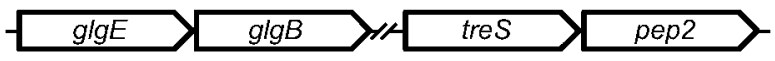

Fig. 4. Clustering of the GlgE pathway genes. Clustering of all four gene functions occurred either (a) with a treS-pep2 fusion (e.g. Pseudomonas aeruginosa) or (b) without (e.g. Streptomyces coelicolor). Another common configuration was clustering in pairs (c) often with more than 1000 genes between the pairs (e.g. Mycobacterium tuberculosis). See supplementary material for details. gene annotations were also taken into account. Thus, it appears to be a rarity for the GlgA and GlgE pathways each to be associated with a dedicated $\operatorname{glg} B$ gene within a single organism.

The Rv3031 gene, predicted to code for the branching enzyme of the Rv3032 pathway (Jackson \& Brennan, 2009), is sufficiently distinct from $\operatorname{glg} B$ that reciprocal BLAST searches do not confuse one with the other. This is a reflection of Rv3031 being a member of the GH57 family rather than the GH13 family that GlgB belongs to, with differences at the structural (Palomo et al., 2011) as well as primary sequence levels (Murakami et al., 2006). This raises the question as to whether a GH57 branching enzyme fulfils the role of $\mathrm{GlgB}$ in some organisms. Reciprocal BLAST searches using Rv3031 as the query revealed that $11 \%$ of genomes possessed a candidate gene for a GH57 branching enzyme, consistent with searches reported by others (Murakami et al., 2006). In $2 \%$ of genomes, the gene was clustered with Rv3030 and Rv3032 (Fig. 3a). However, the presence of a GH57 gene does not correlate with a lack of a $g l g B$ gene (Fig. 3c), so $25 \%$ of genomes that possess glgA are devoid of any known branching enzyme. This could reflect the operation of an unknown kind of branching enzyme, or the production of unbranched glucans. The latter is supported by the transient accumulation of granulose, an $\alpha$-1,4-linked glucan with few or no branches, prior to spore formation in Clostridium acetobutylicum (e.g. Reysenbach et al., 1986). A putative $g l g B$ gene was also present in $3 \%$ of genomes that had none of the three glycosyltransferases, implying still undefined glucan pathways, other roles for $\mathrm{GlgB}$, or the presence of orphaned genes.

The GlgP-mediated regeneration of glucose 1-phosphate could allow glucan cycling or provide activated glucose for other purposes. The $g \lg P$ gene, next to a $g \lg E$-containing cluster in streptomycetes and the $g l g A$-containing cluster in E. coli, was almost equally correlated with $g l g A$ and $g l g E$ (Fig. 3d). The presence of $g l g P$ in the absence of any of the $\alpha$-glucan pathway genes implies either a role in catabolizing $\alpha$-glucans from the environment or the existence of as-yetunidentified $\alpha$-glucan pathways.

There are two potential sources of trehalose for the GlgE pathway: either de novo via GalU-OtsA-OtsB or through recycling of cytosolic $\alpha$-glucan via (TreX)-TreY-TreZ. Comparative genomics of a representative gene for each source, ots $A$ and treY, suggested that both sources coexist in most cases when $g \lg E$ is present (data not shown).

\section{Developmental glycogen/trehalose cycling in streptomycetes}

The developmentally complex soil bacterium S. coelicolor experiences a very different environment to M. tuberculosis. The GlgE pathway genes are duplicated and separately developmentally regulated in S. coelicolor, being associated respectively with transient deposition of glycogen at the 
initiation of aerial growth (phase I) and during the first stages of sporulation (phase II) (Plaskitt \& Chater, 1995; Schneider et al., 2000; Yeo \& Chater, 2005). Trehalose accumulates to high levels in spores at the expense of phase II glycogen deposits (Rueda et al., 2001), contributing significantly to resistance to heat and desiccation (McBride \& Ensign, 1987), and is degraded during spore germination through the activation of a spore-associated trehalase proenzyme (McBride \& Ensign, 1990). The discovery of the function of the GlgE pathway genes now provides an attractive hypothesis for the main metabolic processes underlying these observations. It is possible that the $\mathrm{GlgE}$ pathway is responsible, at least in part (Martin et al., 1997), for the formation of the glycogen deposits in both phases, and that recycling could involve either glucose phosphate or disaccharide intermediates.

\section{The GIgE pathway and non-Mycobacterium pathogens}

Although present in M. tuberculosis and Pseudomonas, Burkholderia and Xanthomonas animal and plant pathogens, the GlgE pathway is absent from most other pathogens. Nevertheless, experimental deletion of GlgE pathway genes and other loci associated with trehalose metabolism lowered Pseudomonas syringae fitness on host and non-host plant leaves (Freeman et al., 2010). Whether this was due to reduced virulence or increased susceptibility to hyperosmotic stress is not known. Interestingly, $g l g E$ appears in the list of genes that have undergone positive selection in the evolution of accidental virulence of Burkholderia pseudomallei (Nandi et al., 2010). The role of the GlgE pathway in pathogens requires further study.

\section{Some unexpected anomalies...}

The activity of phosphoglucomutase PgmA is ostensibly ultimately required to provide glucose 1-phosphate for all routes to the biosynthesis of both $\alpha$-glucan and trehalose. The absence of a pgmA gene from $\sim 11 \%$ of genomes that possess other genes needed for $\alpha$-glucan synthesis is therefore problematical (Fig. 3e), and raises the possibility of either a different way of generating glucose 1-phosphate, or a novel form of phosphoglucomutase that is unrelated to PgmA. By contrast, the presence of pgmA in the absence of $\alpha$-glucan and trehalose pathway genes is to be expected, given the requirement of many metabolites to be glucosidated.

\section{...and some immediate questions}

Additional questions are raised by the existence of three $\alpha$-glucan pathways, often with two or even all three within the same organism. Which of the pathways are primarily responsible for which of the three $\alpha$-glucan products and how much cross-talk is there between the pathways in different contexts? What is the nature of the $\alpha$-glucan produced by the GlgE pathway and how does it compare with cytosolic glycogen and capsular glucan? The comparative genomic analysis supports the notion that the Rv3032 pathway is relatively specific for methylglucose lipopolysaccharide biosynthesis in a limited set of organisms, but evidence for redundancy with the GlgE pathway (Kalscheuer et al., 2010) suggests that one should not view the pathways independently. Could Rv3031 and Rv3032 make a glycogen-like molecule? Furthermore, what is the role of the many Rv3032 glycosyltransferase homologues when not associated with methylglucose lipopolysaccharide biosynthesis? Since GlgB branching enzyme is more often associated with the GlgE pathway than with the classical pathway, are branching enzymes shared between pathways, or is another branching enzyme yet to be discovered? Evidently there is a great deal more to discover about the biology of $\alpha$-glucan metabolism in bacteria, providing new opportunities to tackle some important human and plant pathogens.

\section{Acknowledgements}

This work was supported by the United Kingdom Biotechnology and Biological Sciences Research Council through the MET Institute Strategic Programme Grant to the John Innes Centre. We thank Rainer Kalscheuer, William R. Jacobs, Jr and Alison M. Smith for their comments on the manuscript.

\section{References}

Argüelles, J. C. (2000). Physiological roles of trehalose in bacteria and yeasts: a comparative analysis. Arch Microbiol 174, 217-224.

Belanger, A. E. \& Hatfull, G. F. (1999). Exponential-phase glycogen recycling is essential for growth of Mycobacterium smegmatis. J Bacteriol 181, 6670-6678.

Cantarel, B. L., Coutinho, P. M., Rancurel, C., Bernard, T., Lombard, V. \& Henrissat, B. (2009). The Carbohydrate-Active EnZymes database (CAZy): an expert resource for glycogenomics. Nucleic Acids Res 37 (Database issue), D233-D238.

Carroll, J. D., Pastuszak, I., Edavana, V. K., Pan, Y. T. \& Elbein, A. D. (2007). A novel trehalase from Mycobacterium smegmatis - purification, properties, requirements. FEBS J 274, 1701-1714.

Dinadayala, P., Sambou, T., Daffé, M. \& Lemassu, A. (2008). Comparative structural analyses of the $\alpha$-glucan and glycogen from Mycobacterium bovis. Glycobiology 18, 502-508.

Elbein, A. D., Pan, Y. T., Pastuszak, I. \& Carroll, D. (2003). New insights on trehalose: a multifunctional molecule. Glycobiology 13, 17R-27R.

Elbein, A. D., Pastuszak, I., Tackett, A. J., Wilson, T. \& Pan, Y. T. (2010). Last step in the conversion of trehalose to glycogen: a mycobacterial enzyme that transfers maltose from maltose 1phosphate to glycogen. J Biol Chem 285, 9803-9812.

Freeman, B. C., Chen, C. L. \& Beattie, G. A. (2010). Identification of the trehalose biosynthetic loci of Pseudomonas syringae and their contribution to fitness in the phyllosphere. Environ Microbiol 12, 1486-1497.

Gagliardi, M. C., Lemassu, A., Teloni, R., Mariotti, S., Sargentini, V., Pardini, M., Daffé, M. \& Nisini, R. (2007). Cell wall-associated $\alpha$-glucan is instrumental for Mycobacterium tuberculosis to block CD1 molecule expression and disable the function of dendritic cell derived from infected monocyte. Cell Microbiol 9, 2081-2092. 
Jackson, M. \& Brennan, P. J. (2009). Polymethylated polysaccharides from Mycobacterium species revisited. J Biol Chem 284, 1949-1953.

Jarling, M., Cauvet, T., Grundmeier, M., Kuhnert, K. \& Pape, H. (2004). Isolation of mak1 from Actinoplanes missouriensis and evidence that Pep2 from Streptomyces coelicolor is a maltokinase. J Basic Microbiol 44, 360-373.

Kalscheuer, R., Syson, K., Veeraraghavan, U., Weinrick, B., Biermann, K. E., Liu, Z., Sacchettini, J. C., Besra, G., Bornemann, S. \& Jacobs, W. R., Jr (2010). Self-poisoning of Mycobacterium tuberculosis by targeting GlgE in an $\alpha$-glucan pathway. Nat Chem Biol 6, 376-384.

Kaur, D., Guerin, M. E., Škovierová, H., Brennan, P. J. \& Jackson, M. (2009a). Biogenesis of the cell wall and other glycoconjugates of Mycobacterium tuberculosis. In Advances in Applied Microbiology, pp. 23-77. Edited by A. Laskin, G. Gadd \& S. Sariaslani. Amsterdam: Elsevier.

Kaur, D., Pham, H., Larrouy-Maumus, G., Rivière, M., Vissa, V., Guerin, M. E., Puzo, G., Brennan, P. J. \& Jackson, M. (2009b). Initiation of methylglucose lipopolysaccharide biosynthesis in mycobacteria. PLoS ONE 4, e5447.

Lee, Y. C. (1966). Isolation and characterization of lipopolysaccharides containing 6-O-methyl-D-glucose from Mycobacterium species. J Biol Chem 241, 1899-1908.

Martin, M. C., Schneider, D., Bruton, C. J., Chater, K. F. \& Hardisson, C. (1997). A $g l g C$ gene essential only for the first of two spatially distinct phases of glycogen synthesis in Streptomyces coelicolor A3(2). J Bacteriol 179, 7784-7789.

Maruta, K., Mitsuzumi, H., Nakada, T., Kubota, M., Chaen, H., Fukuda, S., Sugimoto, T. \& Kurimoto, M. (1996). Cloning and sequencing of a cluster of genes encoding novel enzymes of trehalose biosynthesis from thermophilic archaebacterium Sulfolobus acidocaldarius. Biochim Biophys Acta 1291, 177-181.

McBride, M. J. \& Ensign, J. C. (1987). Metabolism of endogenous trehalose by Streptomyces griseus spores and by spores or cells of other actinomycetes. J Bacteriol 169, 5002-5007.

McBride, M. J. \& Ensign, J. C. (1990). Regulation of trehalose metabolism by Streptomyces griseus spores. J Bacteriol 172, 3637-3643.

Murakami, T., Kanai, T., Takata, H., Kuriki, T. \& Imanaka, T. (2006). A novel branching enzyme of the GH-57 family in the hyperthermophilic archaeon Thermococcus kodakaraensis KOD1. J Bacteriol 188, 5915-5924.

Nandi, T., Ong, C., Singh, A. P., Boddey, J., Atkins, T., Sarkar-Tyson, M., Essex-Lopresti, A. E., Chua, H. H., Pearson, T. \& other authors (2010). A genomic survey of positive selection in Burkholderia pseudomallei provides insights into the evolution of accidental virulence. PLoS Pathog 6, e1000845.

Nishimoto, T., Nakano, M., Nakada, T., Chaen, H., Fukuda, S., Sugimoto, T., Kurimoto, M. \& Tsujisaka, Y. (1996). Purification and properties of a novel enzyme, trehalose synthase, from Pimelobacter sp. R48. Biosci Biotechnol Biochem 60, 640-644.

Palomo, M., Pijning, T., Booiman, T., Dobruchowska, J. M., van der Vlist, J., Kralj, S., Planas, A., Loos, K., Kamerling, J. P. \& other authors (2011). Thermus thermophilus glycoside hydrolase family 57 branching enzyme: crystal structure, mechanism of action, and products formed. J Biol Chem 286, 3520-3530.

Pan, Y. T., Koroth Edavana, V., Jourdian, W. J., Edmondson, R., Carroll, J. D., Pastuszak, I. \& Elbein, A. D. (2004). Trehalose synthase of Mycobacterium smegmatis: purification, cloning, expression, and properties of the enzyme. Eur J Biochem 271, 4259-4269.

Plaskitt, K. A. \& Chater, K. F. (1995). Influences of developmental genes on localized glycogen deposition in colonies of a mycelial prokaryote, Streptomyces coelicolor A3(2) - a possible interface between metabolism and mophogenesis. Philos Trans $R$ Soc Lond B Biol Sci 347, 105-121.

Pommier, M. T. \& Michel, G. (1986). Isolation and characterization of an $\mathrm{O}$-methylglucose-containing lipopolysaccharide produced by Nocardia otitidis-caviarum. J Gen Microbiol 132, 2433-2441.

Preiss, J. (2006). Bacterial glycogen inclusions: enzymology and regulation of synthesis. In Microbiology Monographs, pp. 71-108. Edited by J. M. Shively. Heidelberg, Germany: Springer.

Preiss, J. (2009). Glycogen biosynthesis. In The Encyclopedia of Microbiology, vol. 5, pp. 145-158. Edited by M. Schaechter. Oxford, UK: Elsevier.

Preiss, J. \& Romeo, T. (1989). Physiology, biochemistry and genetics of bacterial glycogen synthesis. Adv Microb Physiol 30, 183-238.

Reysenbach, A. L., Ravenscroft, N., Long, S., Jones, D. T. \& Woods, D. R. (1986). Characterization, biosynthesis, and regulation of granulose in Clostridium acetobutylicum. Appl Environ Microbiol 52, 185-190.

Rueda, B., Miguélez, E. M., Hardisson, C. \& Manzanal, M. B. (2001). Changes in glycogen and trehalose content of Streptomyces brasiliensis hyphae during growth in liquid cultures under sporulating and nonsporulating conditions. FEMS Microbiol Lett 194, 181-185.

Sambou, T., Dinadayala, P., Stadthagen, G., Barilone, N., Bordat, Y., Constant, P., Levillain, F., Neyrolles, O., Gicquel, B. \& other authors (2008). Capsular glucan and intracellular glycogen of Mycobacterium tuberculosis: biosynthesis and impact on the persistence in mice. $\mathrm{Mol}$ Microbiol 70, 762-774.

Schneider, D., Bruton, C. J. \& Chater, K. F. (2000). Duplicated gene clusters suggest an interplay of glycogen and trehalose metabolism during sequential stages of aerial mycelium development in Streptomyces coelicolor A3(2). Mol Gen Genet 263, 543-553.

Stadthagen, G., Sambou, T., Guerin, M., Barilone, N., Boudou, F., Korduláková, J., Charles, P., Alzari, P. M., Lemassu, A. \& other authors (2007). Genetic basis for the biosynthesis of methylglucose lipopolysaccharides in Mycobacterium tuberculosis. J Biol Chem 282, 27270-27276.

Takayama, K., Wang, C. \& Besra, G. S. (2005). Pathway to synthesis and processing of mycolic acids in Mycobacterium tuberculosis. Clin Microbiol Rev 18, 81-101.

Yeo, M. \& Chater, K. (2005). The interplay of glycogen metabolism and differentiation provides an insight into the developmental biology of Streptomyces coelicolor. Microbiology 151, 855-861. 